\title{
Risk of lower limb amputation in a national prevalent cohort of patients with diabetes
}

\author{
Jason K. Gurney ${ }^{1}$ (D) James Stanley ${ }^{1} \cdot$ Steve York $^{2} \cdot$ Dieter Rosenbaum $^{3}$ • \\ Diana Sarfati ${ }^{1}$
}

Received: 4 September 2017 / Accepted: 9 October 2017 /Published online: 3 November 2017

(C) Springer-Verlag GmbH Germany 2017

\begin{abstract}
Aims/hypothesis Lower limb amputation is a serious complication of diabetes mellitus. Understanding how amputation risk differs by population subgroups is crucial in terms of directing preventive strategies. In this study, we describe those factors that impact amputation risk in the entire prevalent diabetic population of New Zealand.

Methods A national prevalent cohort of 217,207 individuals with diabetes in 2010 were followed up until the end of 2013 for lower limb amputations, and 2014 for mortality. Inpatient hospitalisation data were used to define lower limb amputation using ICD-10 codes. Cox proportional hazards models were used to describe relative hazard of amputation over the follow-up period.

Results A total of 784 individuals (3.6 cases/1000 individuals) underwent a major (above-ankle) lower limb amputation during follow-up, while 1217 (5.6/1000) underwent a minor (below ankle) amputation. The risk of major and minor amputation was $39 \%$ and $77 \%$ greater for men than women, respectively (adjusted HR: major amputation 1.39, 95\% CI 1.20, 1.61; minor
\end{abstract}

Electronic supplementary material The online version of this article (https://doi.org/10.1007/s00125-017-4488-8) contains peer-reviewed but unedited supplementary material, which is available to authorised users.

Jason K. Gurney

jason.gurney@otago.ac.nz

1 Cancer and Chronic Conditions (C3) Research Group, Department of Public Health, University of Otago, 23a Mein St, Newtown, Wellington, New Zealand

2 High Risk Foot Clinic, Northland District Health Board, Whangarei, New Zealand

3 Movement Analysis Lab, University Hospital Muenster, Muenster, Germany amputation 1.77, 95\% CI 1.56, 2.00). Indigenous Māori were at $65 \%$ greater risk of above-knee amputation compared with the European/Other diabetic population (HR 1.65, 95\% CI 1.37, 1.97). Amputation risk increased with increasing comorbidity burden, and peripheral vascular disease conferred the greatest independent risk of all comorbid conditions. Prior minor amputation increased the risk of subsequent major amputation by tenfold (HR 10.04, 95\% CI 7.83, 12.87), and increased the risk of another minor amputation by 20 -fold (HR $21.39,95 \%$ CI $17.89,25.57)$. Death was common among the total cohort, but particularly among those who underwent amputation, with more than half of those who underwent a major amputation dying within 3 years of their procedure $(57 \%)$.

Conclusions/interpretation Using a large, well-defined, national prevalent cohort of people with diabetes, we found that being male, indigenous Māori, living in deprivation, having a high comorbidity burden and/or having a previous amputation were strongly associated with subsequent risk of lower limb amputation. The use of this prevalent cohort strengthens the value of our estimates in terms of applicability to the general population, and highlights the subgroups at greatest risk of lower limb amputation.

Keywords Amputation - Comorbidity · Deprivation . Diabetes mellitus $\cdot$ Ethnicity $\cdot$ Lower limb $\cdot$ Prevalent cohort

$\begin{array}{ll}\text { Abbreviations } \\ \text { NZDep } & \text { New Zealand Deprivation Index } \\ \text { URPC } & \text { Urban/Rural Profile Classification } \\ \text { VDR } & \text { Virtual Diabetes Register }\end{array}$

\section{Introduction}

There are many factors that contribute to risk of amputation among people with diabetes [1]. At a fundamental level, poor 
control of glucose and vascular health over the course of multiple preceding years is an important catalyst [2-7]. Subsequent poor management of peripheral neuropathy and peripheral vascular disease are intermediary contributors to amputation risk, while subsequent poor management of foot wounds/ulcers (including offloading) — combined with poor healing capacity [8], itself driven by poor vascular and autonomic function - can impact amputation risk in a more proximal sense. Those individuals who have had a prior amputation are also at substantially increased risk of subsequent amputation [9-11], which is likely driven by advanced peripheral vascular and neuropathic disease [10]. Previous reviews have reported high levels of both short-term and long-term mortality among individuals with diabetes who undergo amputation $[12,13]$.

The disease factors that contribute to amputation risk are not evenly distributed among diabetic populations. A cohort study of 62,000 individuals attending annual diabetes checks in New Zealand found that those people with diabetes living in the most-deprived areas were $50 \%$ more likely to require amputation (adjusted HR 1.51, 95\% CI 1.15, 1.97), while women were half as likely to require amputation compared with men (adjusted HR 0.48, 95\% CI 0.42, 0.55) [14]. The latter finding has been repeatedly observed in other international contexts [9, 15-18]. However, it is common for studies of amputation risk to be conducted among those regularly attending diabetes check-ups - the large New Zealand study was conducted among those attending annual diabetes reviews, which is estimated to be fewer than half of the total diabetic population [14]; as such, it is difficult to know if these observations are applicable to the wider population of all people with diabetes. It also remains unclear whether these disparities might be wholly or partially explained by strong known risk factors, including comorbidity (particularly peripheral vascular disease) and prior amputation.

Understanding how amputation risk differs by population subgroups is crucial for directing preventive strategies [19]. Therefore, the objective of this study was to describe those factors that impact the risk of amputation among all prevalent diabetic individuals in New Zealand, stratified by population subgroup and with separate analyses for major and minor amputations.

\section{Methods}

\section{Participants and data sources}

A prevalent cohort of 217,207 individuals with diabetes in 2010 were followed until the end of 2013 for lower limb amputations, and 2014 for mortality. The prevalent cohort of individuals with diabetes was defined using the New Zealand Ministry of Health's Virtual Diabetes Register (VDR), which uses information from multiple national-level data sources to attribute a diagnosis of diabetes to a given individual. The methods underlying the VDR are detailed in ESM Methods and are based on relevant inpatient and outpatient activity, pharmaceutical prescriptions and laboratory tests [20]. The VDR is used to determine official diagnosed diabetes prevalence in New Zealand [21]; however, in contrast to the approach used by the Ministry of Health we have included individuals who could not be linked to a primary health organisation, as well as those who died before the end of 2010. Once this cohort was defined, each individual was further linked to the following datasets.

Inpatient hospitalisation records Each individual was linked to all hospitalisation records from 2005 until 2013. Using this dataset, we: (1) identified all lower limb amputations that occurred between 2010 and 2013 using ICD-10 codes (www. who.int/classifications/icd/en/) (see ESM Table 1); (2) identified amputations that occurred during the 5 year period prior to the study period (i.e. 2005-2009) using the same codes; and (3) identified comorbid conditions diagnosed in the 5 years prior to 2010 .

Mortality records, 2005-2014 Each individual in the cohort was linked to mortality records from 2005 to the end of 2014 .

\section{Variables}

Demographic variables for all individuals, including age, sex, ethnicity, deprivation and rurality, were determined using data from the VDR.

Age was defined as age in years as of the beginning of the study period (i.e. 1 January 2010) and further categorised into five age groups $(<25,25-49,50-64,65-75, \geq 75)$. Sex was defined as either male or female. Ethnicity was defined as Māori, Pacific, Asian or European/Other (non-Māori/Pacific/ Asian). Each individual was attributed an index date based on the beginning of the study period (1 January 2010).

Level of patient deprivation was determined using New Zealand's Deprivation Index (NZDep) [22], a small-area based index calculated using aggregated census data based on socioeconomic characteristics of the area in which an individual lives and ranked into quintiles ranging between 1 (least deprived) and 5 (most deprived). Rurality was defined using a modified version of the Urban/Rural Profile Classification (URPC) [23], a categorisation system which allows mapping of New Zealand Census Area Unit data down to three classifications: urban (main urban area + satellite urban area); independent urban area; and rural (all rural areas). Deprivation and/or rurality could not be determined for $n=2281$ individuals ( $1.0 \%$ of the total cohort) because of missing data.

Comorbidity Comorbidity was defined using the Charlson comorbidity index, which employs inpatient hospitalisation 
data to define the presence of chronic conditions in addition to the primary condition (i.e. diabetes) [24, 25]. Diabetes with and without complications were excluded from the score calculation. Using this index, a comorbidity score was assigned to each individual based on ICD codes recorded during inpatient hospitalisations during the 5 years prior to the index date (1 January 2005-31 December 2009). Scores were categorised as 0 (no comorbidity), 1, 2 and 3+ (severe comorbidity). If individuals had no hospitalisation data for the 5 year lookback period, their comorbidity score was set at 0 .

Lower limb amputation During follow-up, lower limb amputation was defined using inpatient hospitalisation procedure ICD codes (ESM Table 1). Amputations were categorised as either major (above or through ankle) or minor (below ankle) [26]. The first amputation of each type was noted for each given individual as a binary variable (yes/no) recording whether an amputation occurred during the follow-up period, and a date variable recording the date of the amputation. It was possible for a given individual to be recorded as having both a major and minor amputation, but only one of each type (i.e. the earliest during the followup period) was recorded for each person. We also looked for prior amputations that occurred in the 5 year period before the study start date (1 January 2005-31 December 2009); here again, we searched for both major amputations and minor amputations (it is possible that a given individual could have both). Again, a binary variable (yes/no) recorded whether one or both of the amputation types occurred during the lookback period. Mortality was defined using date of death data from the national mortality collection.

This study assumes that amputations occurring within our diabetic cohort are related to their diabetes and associated complications, rather than other unrelated factors. To assess the impact of this assumption, we investigated the number of amputations over the follow-up period that had lower limb cancer and/or trauma ICD codes listed within the same hospital event as the amputation. Only one amputation patient $(<0.1 \%)$ had a trauma code within the same hospital event as the amputation procedure, and no amputation individuals had a lower limb cancer code at time of amputation.

\section{Statistical analysis}

Descriptive analyses Crude descriptive analyses were used to describe the demographic characteristics of the cohort and the occurrence of lower limb amputation, stratified by demographic characteristics and comorbidity. We also described the prevalence of individual comorbid conditions at baseline for both those who had an amputation and those who did not.

Risk of amputation Crude and adjusted risk of major or minor lower limb amputation was investigated using Cox proportional hazards models. Individuals were followed for a 4 year period from 1 January 2010 until 31 December 2013. Individuals were censored at the date of their death if they died during the follow-up period.

Separate models were constructed for each demographic covariate as the exposure of interest (i.e. crude estimates), along with fully adjusted models which included all other covariates. All variables were included in the models as categorical variables. HRs and their $95 \%$ CIs were determined from the models.

Finally, we calculated the crude and adjusted risk of amputation (HRs and 95\% CIs) for each individual Charlson comorbid condition (excluding the two diabetes-related conditions). For the fully adjusted model, we included sex, age, ethnicity, deprivation, rurality and prior amputation as categorical covariates.

Risk of death We compared the crude risk of death between amputation groups over the study period using crude descriptive and Kaplan-Meier analysis. The start of the follow-up period for those who underwent an amputation was the date of their amputation, while the start of the follow-up period for those who did not undergo an amputation was the beginning of the study period (1 January 2010). We determined the number and proportion of deaths within each amputation group, and also for those who did not undergo an amputation. We then calculated 1, 2 and 3 year post-amputation mortality rates for those who underwent a minor amputation only (i.e. excluding those who underwent a major amputation), as well as those who underwent a major amputation. Individuals were censored at the end of follow-up if they did not die during the study.

\section{Ethics approval}

This study received ethical approval from the University of Otago Human Ethics Committee Health (reference HD16/055).

\section{Results}

Of the 217,207 individuals with diabetes identified from the VDR (Table 1), a total of 784 individuals (3.6 cases/1000 individuals) underwent a major amputation, while 1217 underwent a minor amputation (5.6 cases/1000; Table 2). Major and minor amputations were more common among men (major amputation: 4.4 cases/1,000 men; 2.7 cases/ 1,000 women; minor amputation: 7.4 cases/1,000 men; 3.7 cases $/ 1,000$ women). The mean age of those requiring major amputation (67.1 years [SD 13 years]) was marginally higher than those requiring minor amputation (65.0 years [SD: 13.3 years]; Table 2 ). 
Table 1 Demographic and comorbid characteristics of the 2010 prevalent diabetic population of New Zealand

\begin{tabular}{|c|c|c|}
\hline \multirow[t]{2}{*}{ Characteristic } & \multicolumn{2}{|c|}{ Total cohort } \\
\hline & $n$ & $\%$ \\
\hline Total cohort ${ }^{\mathrm{a}}$ & 217,207 & - \\
\hline \multicolumn{3}{|l|}{ Sex } \\
\hline Female & 105,987 & 48.8 \\
\hline Male & 111,219 & 51.2 \\
\hline \multicolumn{3}{|l|}{ Age (years) ${ }^{\mathrm{b}}$} \\
\hline $0-24$ & 5321 & 2.5 \\
\hline $25-49$ & 40,794 & 18.8 \\
\hline $50-64$ & 69,863 & 32.2 \\
\hline $65-74$ & 49,278 & 22.7 \\
\hline$\geq 75$ & 51,951 & 23.9 \\
\hline Mean & 62.5 & 16.6 \\
\hline \multicolumn{3}{|l|}{ Ethnicity } \\
\hline European/Other & 137,445 & 63.3 \\
\hline Māori & 31,249 & 14.4 \\
\hline Pacific & 25,592 & 11.8 \\
\hline Asian & 22,921 & 10.6 \\
\hline \multicolumn{3}{|l|}{ Deprivation (NZDep) } \\
\hline 1-2 (least deprived) & 26,679 & 12.4 \\
\hline $3-4$ & 31,929 & 14.9 \\
\hline $5-6$ & 38,627 & 18.0 \\
\hline $7-8$ & 51,123 & 23.8 \\
\hline 9-10 (most deprived) & 66,718 & 31.0 \\
\hline \multicolumn{3}{|l|}{ Rurality (URPC) } \\
\hline Urban & 163,581 & 76.1 \\
\hline Ind. urban & 30,081 & 14.0 \\
\hline Rural & 21,269 & 9.9 \\
\hline \multicolumn{3}{|l|}{ Comorbidity (Charlson) } \\
\hline 0 & 172,742 & 79.5 \\
\hline 1 & 17,100 & 7.9 \\
\hline 2 & 11,708 & 5.4 \\
\hline $3+$ & 15,657 & 7.2 \\
\hline \multicolumn{3}{|l|}{ Prior amputation } \\
\hline Major & 545 & 0.3 \\
\hline Minor & 937 & 0.4 \\
\hline
\end{tabular}

Those with missing data are excluded from the denominator when calculating proportions

${ }^{\text {a }}$ As determined from the VDR

${ }^{\mathrm{b}}$ Age at start of follow-up period (1 January 2010) Ind., independent

Major amputation risk during the follow-up period was greatest among Māori (6.4 cases/1,000 Māori individuals) followed by European/Other (3.6 cases/1,000 European/ Other individuals), Pacific (2.7 cases/1,000 Pacific individuals) and Asian individuals (1.1 cases/1,000 Asian individuals). The trend was similar for minor amputations. The risk of major and minor amputation was greatest among those
Table 2 Major and minor lower limb amputation among the cohort, by demographic characteristic

\begin{tabular}{|c|c|c|c|c|}
\hline \multirow[t]{3}{*}{ Characteristic } & \multicolumn{4}{|c|}{ Amputations } \\
\hline & \multicolumn{2}{|c|}{ Major } & \multicolumn{2}{|c|}{ Minor } \\
\hline & $n^{\mathrm{a}}$ & $n / 1000$ & $\mathrm{n}^{\mathrm{a}}$ & $n / 1000$ \\
\hline Total amputations & 784 & 3.6 & 1217 & 5.6 \\
\hline \multicolumn{5}{|l|}{ Sex } \\
\hline Female & 290 & 2.7 & 389 & 3.7 \\
\hline Male & 494 & 4.4 & 828 & 7.4 \\
\hline \multicolumn{5}{|l|}{ Age (years) } \\
\hline $0-24$ & 2 & 0.4 & 1 & 0.2 \\
\hline $25-49$ & 75 & 1.8 & 176 & 4.3 \\
\hline $50-64$ & 250 & 3.6 & 413 & 5.9 \\
\hline $65-74$ & 213 & 4.3 & 317 & 6.4 \\
\hline$\geq 75$ & 244 & 4.7 & 310 & 6.0 \\
\hline Mean & 67.1 & 13.0 & 65 & 13.3 \\
\hline \multicolumn{5}{|l|}{ Ethnicity } \\
\hline European/Other & 489 & 3.6 & 815 & 5.9 \\
\hline Māori & 201 & 6.4 & 221 & 7.1 \\
\hline Pacific & 69 & 2.7 & 141 & 5.5 \\
\hline Asian & 25 & 1.1 & 40 & 1.7 \\
\hline \multicolumn{5}{|l|}{ Deprivation (NZDep) } \\
\hline $1-2$ & 58 & 2.2 & 123 & 4.6 \\
\hline $3-4$ & 102 & 3.2 & 142 & 4.4 \\
\hline $5-6$ & 131 & 3.4 & 203 & 5.3 \\
\hline $7-8$ & 197 & 3.9 & 321 & 6.3 \\
\hline $9-10$ & 295 & 4.4 & 424 & 6.4 \\
\hline \multicolumn{5}{|l|}{ Rurality (URPC) } \\
\hline Urban & 575 & 3.5 & 897 & 5.5 \\
\hline Ind. urban & 137 & 4.6 & 189 & 6.3 \\
\hline Rural & 71 & 3.3 & 126 & 5.9 \\
\hline \multicolumn{5}{|l|}{ Comorbidity (Charlson) } \\
\hline 0 & 240 & 1.4 & 527 & 3.1 \\
\hline 1 & 108 & 6.3 & 172 & 10.1 \\
\hline 2 & 110 & 9.4 & 153 & 13.1 \\
\hline $3+$ & 326 & 20.8 & 365 & 23.3 \\
\hline \multicolumn{5}{|l|}{ Prior amputation } \\
\hline Major & 69 & 126.6 & 35 & 37.4 \\
\hline Minor & 121 & 222.0 & 189 & 201.7 \\
\hline
\end{tabular}

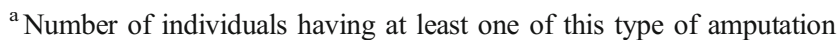
over the follow-up period (2010-2013) Ind., independent

residing in the two most-deprived quintiles, and the risk of major or minor amputation was similar regardless of urban/ rural status. Major and minor amputation risk increased substantially with increasing comorbidity burden (e.g. major amputation: Charlson score $0,1.4$ cases/1,000 individuals; Charlson score 1, 6.3 cases/1,000; Charlson score 2, 9.4 cases/1,000; Charlson score 3+, 20.8 cases/1,000). Around 
$13 \%$ of those who had a prior major amputation in the five years prior to the study period underwent a subsequent major amputation during the follow-up period ( $n=69,126.6$ cases/ 1,000 individuals). Around $22 \%$ of those who had undergone a prior minor amputation underwent a subsequent major amputation ( $n=121,222$ cases/1,000 individuals), while $20 \%$ underwent a subsequent minor amputation $(n=189,201.7$ cases/1,000 individuals; Table 2).

Crude and adjusted HRs comparing the risk of amputation during the follow-up period by demographic and comorbid covariates are shown in Table 3 . We observed that the risk of major amputation among men was nearly $40 \%$ greater than it was for women (adjusted HR 1.39, 95\% CI 1.20, 1.61), and risk of minor amputation was nearly $80 \%$ greater for men (1.77, 95\% CI 1.56, 2.00; Table 3).

While crude estimates suggested that younger age was protective against major amputation risk (crude HR 50-64 years $0.68,95 \%$ CI $0.57,0.82$; reference group is $\geq 75$ ), this difference was largely accounted for after adjusting for covariates, including comorbidity (adjusted HR 0.89, 95\% CI 0.74, 1.07). A similar trend was observed for minor amputations (Table 3 ).

The risk of major amputation among Māori individuals was substantially higher than for European/Other individuals (adjusted HR 1.65, 95\% CI 1.37, 1.97); however, Māori were at no more risk of minor amputation $(1.06$, 95\% CI 0.90 , 1.25). Pacific individuals appeared at lower risk of both major $(0.73,95 \%$ CI $0.55,0.95)$ and minor $(0.85,95 \%$ CI $0.70,1.03)$ amputation than European/Other individuals, while Asian individuals were at a substantially reduced risk of both major $(0.43,95 \%$ CI $0.29,0.65)$ and minor $(0.36,95 \%$ CI $0.26,0.50$; Table 3) amputation.

Risk of amputation during the follow-up period appeared to decrease at lower levels of deprivation; for example, those residing in the least-deprived deciles experienced $34 \%$ lower risk of major amputation relative to the most deprived (NZDep quintile 5=reference, NZDep quintile 1, adjusted HR 0.66, $95 \%$ CI $0.49,0.88)$. Amputation risk did not seem to substantially differ based on urban or rural residence (Table 3 ).

Comorbidity had a profound impact on risk of amputation. Compared with those with no significant comorbidity (Charlson score category 0), individuals in Charlson category 1 were at nearly four times greater risk of major amputation (adjusted HR 3.83, 95\% CI 3.05, 4.82), while individuals in Charlson category 2 had more than six times the risk $(6.04$, $95 \%$ CI $4.80,7.60)$ and those in Charlson category $3+$ had more than 12 times the risk $(12.31,95 \%$ CI 10.26, 14.77). Similar trends were observed for minor amputations, although the magnitude of the HRs was lower (Table 3).

Of all the variables under consideration, prior amputation conferred the strongest increase in amputation risk. Prior major amputation increased the risk of subsequent major amputation during the follow-up period threefold (adjusted HR 3.06, 95\% CI 2.24, 4.18), while prior minor amputation increased the risk
Table 3 Adjusted instantaneous risk of major and minor amputation in the cohort, by demographic characteristic

\begin{tabular}{|c|c|c|}
\hline \multirow[t]{2}{*}{ Characteristic } & \multicolumn{2}{|l|}{ Amputations } \\
\hline & $\begin{array}{l}\text { Major } \\
\text { Adj. HR (95\% CI) }\end{array}$ & $\begin{array}{l}\text { Minor } \\
\text { Adj. HR }(95 \% \mathrm{CI})^{\mathrm{a}}\end{array}$ \\
\hline \multicolumn{3}{|l|}{ Sex } \\
\hline Female & Reference & Reference \\
\hline Male & $1.39(1.20,1.61)$ & $1.77(1.56,2.00)$ \\
\hline \multicolumn{3}{|l|}{ Age (years) } \\
\hline $0-24$ & $0.17(0.04,0.68)$ & $0.05(0.01,0.38)$ \\
\hline $25-49$ & $0.62(0.48,0.82)$ & $1.08(0.89,1.32)$ \\
\hline $50-64$ & $0.89(0.74,1.07)$ & $1.12(0.95,1.30)$ \\
\hline $65-74$ & $0.86(0.72,1.04)$ & $1.05(0.89,1.23)$ \\
\hline$\geq 75$ & Reference & Reference \\
\hline \multicolumn{3}{|l|}{ Ethnicity } \\
\hline European/Other & Reference & Reference \\
\hline Māori & $1.65(1.37,1.97)$ & $1.06(0.90,1.25)$ \\
\hline Pacific & $0.73(0.55,0.95)$ & $0.85(0.70,1.03)$ \\
\hline Asian & $0.43(0.29,0.65)$ & $0.36(0.26,0.50)$ \\
\hline \multicolumn{3}{|l|}{ Deprivation (NZDep) } \\
\hline $1-2$ & $0.66(0.49,0.88)$ & $0.85(0.68,1.05)$ \\
\hline $3-4$ & $0.90(0.71,1.15)$ & $0.79(0.65,0.97)$ \\
\hline $5-6$ & $0.84(0.68,1.05)$ & $0.88(0.74,1.05)$ \\
\hline $7-8$ & $0.93(0.77,1.12)$ & $1.03(0.88,1.19)$ \\
\hline $9-10$ & Reference & Reference \\
\hline \multicolumn{3}{|l|}{ Rurality (URPC) } \\
\hline Urban & Reference & Reference \\
\hline Ind. urban & $1.01(0.83,1.22)$ & $0.97(0.82,1.14)$ \\
\hline Rural & $0.91(0.7,1.17)$ & $1.07(0.88,1.29)$ \\
\hline \multicolumn{3}{|c|}{ Comorbidity (Charlson) } \\
\hline 0 & Reference & Reference \\
\hline 1 & $3.83(3.05,4.82)$ & $2.83(2.37,3.37)$ \\
\hline 2 & $6.04(4.80,7.60)$ & $3.87(3.22,4.66)$ \\
\hline $3+$ & $12.31(10.26,14.77)$ & $6.63(5.72,7.70)$ \\
\hline \multicolumn{3}{|l|}{ Prior amputation } \\
\hline Major & $3.06(2.24,4.18)$ & $0.61(0.42,0.88)$ \\
\hline Minor & $10.04(7.83,12.87)$ & $21.39(17.89,25.57)$ \\
\hline
\end{tabular}

${ }^{\text {a }}$ Adjusted for all other covariates. Crude estimates are presented in ESM Table 2

Adj., adjusted; Ind., independent

of major amputation by more than ten times $(10.04,95 \% \mathrm{CI}$ $7.83,12.87)$. Prior major amputation reduced the risk of subsequent minor amputation during the follow-up period by nearly $40 \%(0.61,95 \%$ CI $0.42,0.88)$, while prior minor amputation increased the risk of subsequent minor amputation more than 20-fold (21.39, 95\% CI 17.89, 25.57; Table 3).

In terms of individual comorbidity (Table 4), those conditions that were both common among those requiring major amputation ( $>10 \%$ prevalence) and conferred the strongest 
Table 4 Crude and adjusted instantaneous risk of major and minor amputation, by individual comorbidity

\begin{tabular}{|c|c|c|c|c|c|c|c|}
\hline \multirow[b]{3}{*}{ Comorbidity } & \multirow{3}{*}{$\begin{array}{l}\text { No amputation } \\
\text { Prevalence } \\
\mathrm{n}(\%)\end{array}$} & \multicolumn{3}{|c|}{ Major amputation } & \multicolumn{3}{|c|}{ Minor amputation } \\
\hline & & \multirow{2}{*}{$\begin{array}{l}\text { Prevalence } \\
n(\%)\end{array}$} & \multicolumn{2}{|l|}{$\mathrm{HR}(95 \% \mathrm{CI})$} & \multirow{2}{*}{$\begin{array}{l}\text { Prevalence } \\
n(\%)\end{array}$} & \multicolumn{2}{|l|}{ HR $(95 \%$ CI) } \\
\hline & & & Crude & Adjusted $^{\mathrm{a}}$ & & Crude & Adjusted $^{\mathrm{a}}$ \\
\hline Myocardial infarction & $10,822(5.0)$ & $148(18.9)$ & $4.98(4.16,5.95)$ & $3.13(2.60,3.77)$ & $186(15.3)$ & $3.86(3.30,4.52)$ & $2.60(2.22,3.06)$ \\
\hline $\begin{array}{l}\text { Congestive } \\
\text { heart failure }\end{array}$ & $9983(4.6)$ & $179(22.8)$ & $7.74(6.55,9.15)$ & $4.17(3.49,5.00)$ & $208(17.1)$ & $5.40(4.65,6.27)$ & $3.23(2.75,3.79)$ \\
\hline $\begin{array}{l}\text { Peripheral vascular } \\
\text { disease }\end{array}$ & $5373(2.5)$ & $283(36.1)$ & $25.24(21.81,29.21)$ & $12.72(10.62,15.24)$ & $321(26.4)$ & $16.10(14.17,18.30)$ & $7.22(6.09,8.56)$ \\
\hline $\begin{array}{l}\text { Cerebrovascular } \\
\text { disease }\end{array}$ & 8378 (3.9) & $100(12.8)$ & $4.22(3.42,5.20)$ & $2.51(2.02,3.13)$ & $108(8.9)$ & $2.81(2.31,3.42)$ & $1.97(1.61,2.42)$ \\
\hline Dementia & $2126(1.0)$ & $16(2.0)$ & $3.41(2.08,5.60)$ & $2.13(1.29,3.53)$ & $11(0.9)$ & $1.49(0.82,2.70)$ & $1.13(0.62,2.06)$ \\
\hline $\begin{array}{l}\text { Chronic pulmonary } \\
\text { disease }\end{array}$ & $8250(3.8)$ & $70(8.9)$ & $2.85(2.23,3.64)$ & $1.96(1.53,2.52)$ & $73(6.0)$ & $1.86(1.47,2.35)$ & $1.52(1.20,1.93)$ \\
\hline Rheumatic disease & $1048(0.5)$ & $14(1.8)$ & $4.19(2.47,7.11)$ & $2.38(1.39,4.06)$ & $21(1.7)$ & $4.06(2.64,6.25)$ & $2.72(1.76,4.22)$ \\
\hline Peptic ulcer disease & $1821(0.9)$ & $16(2.0)$ & $2.75(1.68,4.52)$ & $1.57(0.95,2.59)$ & $27(2.2)$ & $3.01(2.05,4.40)$ & $2.13(1.45,3.13)$ \\
\hline Liver disease: mild & $1739(0.8)$ & $15(1.9)$ & $2.52(1.51,4.20)$ & $1.92(1.15,3.21)$ & $28(2.3)$ & $3.05(2.10,4.43)$ & $2.31(1.59,3.37)$ \\
\hline $\begin{array}{l}\text { Liver disease: } \\
\text { mod./severe }\end{array}$ & $532(0.3)$ & $4(0.5)$ & - & - & $6(0.5)$ & $2.43(1.09,5.42)$ & $1.67(0.75,3.74)$ \\
\hline Hemi/paraplegia & $4525(2.1)$ & $71(9.1)$ & $5.46(4.27,6.96)$ & $3.18(2.47,4.10)$ & $49(4.0)$ & $2.29(1.72,3.05)$ & $1.61(1.21,2.16)$ \\
\hline Renal disease & $11,083(5.1)$ & $290(37.0)$ & $13.01(11.25,15.05)$ & $7.36(6.24,8.68)$ & $359(29.5)$ & $9.31(8.22,10.53)$ & $5.50(4.77,6.33)$ \\
\hline $\begin{array}{l}\text { Cancer: primary } \\
\text { tumour }\end{array}$ & $7102(3.3)$ & $58(7.4)$ & $2.80(2.14,3.66)$ & $2.16(1.65,2.84)$ & $59(4.8)$ & $1.79(1.38,2.32)$ & $1.46(1.12,1.90)$ \\
\hline $\begin{array}{l}\text { Cancer: secondary } \\
\text { tumour }\end{array}$ & $1724(0.8)$ & $5(0.6)$ & $1.13(0.47,2.72)$ & $1.08(0.45,2.60)$ & $6(0.5)$ & $0.87(0.39,1.95)$ & $0.99(0.44,2.21)$ \\
\hline AIDS/HIV & $19(0.0)$ & $1(0.1)$ & - & - & $1(0.1)$ & - & - \\
\hline
\end{tabular}

HRs not reported where condition prevalence among those having amputation during the follow-up period was lower than $n=5$

Those with missing data are excluded from the denominator when calculating proportions

${ }^{a}$ Adjusted for sex, age, ethnicity, deprivation, rurality and prior amputation

Mod., moderate

increase in major amputation risk included peripheral vascular disease (adjusted HR 12.72, 95\% 10.62, 15.24), renal disease (7.36, 95\% CI 6.24, 8.68), congestive heart failure $(4.17,95 \%$ CI $3.49,5.00)$, myocardial infarction $(3.13,95 \%$ CI 2.60 , $3.77)$ and cerebrovascular disease $(2.51,95 \%$ CI $2.02,3.13)$. Similar trends were observed for minor amputations, although the magnitude of the hazard ratios was lower (Table 3).

Mortality was common among the total cohort: 27,495 of the 217,207 who were included in the study died before the end of the mortality follow-up period (13\% of the cohort). The post-amputation rate of mortality was greatest for those who underwent a major amputation (3 year mortality rate: $57 \%$ ), and also high among those who underwent a minor amputation (35\%; Table 5).

\section{Discussion}

The objective of this paper was to describe those factors that impact the risk of amputation and mortality among a prevalent cohort of individuals with diabetes in New Zealand, stratified by population subgroups and extent of amputation. Our key observations are discussed below, beneath subgroup headings.

\section{Sex}

Men were 40-70\% more likely to have major and minor amputations than women (adjusted HRs 1.39 and 1.77, respectively). The disparity in amputation risk between the sexes has been documented by other investigators in a number of other population contexts $[9,15-18]$. The reason(s) for this disparity remain unclear, but are thought to include a reduced likelihood among men to seek timely diabetic foot care compared with women [17], and are also likely to be driven by the higher risk of vascular disease and smoking among men.

\section{Ethnicity}

We observed that Māori were at no increased risk of minor amputation than the European/Other population, but were at $65 \%$ greater risk of major amputation. This observation is similar to those in other international contexts, where 
Table 5 Total deaths and postamputation mortality rate by amputation group

\begin{tabular}{lllll}
\hline Amputation group & Total deaths & & \multicolumn{2}{l}{ Post-amputation mortality rate $(\%)^{\mathrm{b}}$} \\
\cline { 3 - 4 } & $n(\%)$ & 1 year & 2 years & 3 years \\
\hline No amputation $^{\mathrm{c}}$ & $27,495(13.0)$ & & & \\
Minor amputation $^{\mathrm{d}}$ & $320(34.0)$ & 18.0 & 26.0 & 35.0 \\
Major amputation & $441(56.0)$ & 36.0 & 47.0 & 57.0 \\
\hline
\end{tabular}

Amputation group: 0, no amputation; 1, minor amputation only; 2, major amputation. Kaplan-Meier curves are shown in ESM Fig. 1

${ }^{\mathrm{a}}$ In 2010-2014

${ }^{\mathrm{b}}$ Determined using Kaplan-Meier survival analysis methods

${ }^{\mathrm{c}}$ No amputation during the follow-up period

${ }^{\mathrm{d}}$ Excluding those who also sustained a major amputation indigenous individuals with diabetes have been observed to have higher amputation rates than non-indigenous individuals with diabetes $[27,28]$. Our observation of substantially increased risk of major amputation among Māori, even after adjusting for any differences in factors such as comorbidity and deprivation, is of concern, and requires further investigation.

In contrast to our observations regarding Māori, we observed that Pacific peoples with diabetes were at reduced risk of amputation compared with the European/Other population (adjusted HRs: major amputation 0.73, minor amputation 0.85 ). This unusual observation requires further investigation. Similar to previous authors $[3,14,29]$, we observed that Asian people with diabetes had by far the lowest risk of amputation (adjusted HRs: major amputation 0.43, minor amputation 0.36). Possible explanatory factors for the low risk among Asian individuals might include the low prevalence of peripheral vascular disease, and/or high-quality glycaemic control and complication management among this population [29].

\section{Comorbidity}

We observed a strong association between increasing level of comorbidity and increasing risk of amputation. Those with the most severe comorbidity burden, as measured with the Charlson comorbidity index, were at 12 times greater risk of major amputation and nearly seven times greater risk of minor amputation, even after adjusting for all other covariates. Perhaps unsurprisingly, the individual condition with the strongest association was peripheral vascular disease, which independently increased the risk of major amputation by nearly 13 times, and the risk of minor amputation by more than seven times. This observation largely echoes that found by other authors $[9,10,15,30]$.

It is important to note that many of the conditions that are included in the Charlson index may reflect diabetic severity. Peripheral vascular disease is a key pathway through which diabetes causes lower limb amputation and, as such, conceptually this condition is less a comorbidity than a marker of disease progression and amputation risk. However, it should be noted that peripheral vascular disease is a broad condition, and is not synonymous with peripheral arterial disease of the lower extremity. Other 'comorbidities' including those associated with macrovascular disease (myocardial infarction, stroke, congestive heart failure, etc.) and renal disease are also markers of diabetes severity and could be conceptualised as complications of diabetes. Chronic obstructive pulmonary disorder is a strong marker of smoking status [31], and smoking is, in turn, a strong risk factor for peripheral vascular disease [32]. Comorbidity in this context can be conceptualised as representing three different constructs: (1) conditions that reflect severity of diabetes; (2) conditions that are co-existing risk factors for peripheral vascular disease (and thus amputation); and (3) comorbid conditions per se, which may have an independent impact on likelihood of amputation. It is difficult to disentangle these three, and all are important.

\section{Prior amputation}

Prior amputation was consistently found to be a very strong risk factor for subsequent amputation. We observed a very strong association between prior amputation and risk of subsequent major amputation during follow-up (adjusted HR: prior major amputation $3.06,95 \%$ CI $2.24,4.18$; prior minor amputation $10.04,95 \%$ CI $7.83,12.87)$. Considering the likelihood that more than half of healed diabetic foot ulcers reoccur after 3 years [33], coupled with the likelihood that the individual will likely still have the same risk factors that were key causes of the prior amputation (e.g. peripheral vascular disease), the association between prior amputation and subsequent amputation is not surprising and is in line with findings from other contexts [9-11, 34]. The reasons that have been suggested for this heightened risk include inadequate surgical margins during an original amputation, in an effort to save a greater part of the lower extremity [34], as well as altered biomechanics resulting from the prior amputation leading to 
changes in lower-extremity loading and the development of new bony deformities [11, 35, 36].

The relationship between prior amputation and risk of future amputation may have less to do with the prior amputation per se than it does with the individual's underlying risk factors. The crude (i.e. unadjusted) association between prior amputation and subsequent amputation was remarkable; HRs of around 50 were observed across most amputation types. However, this association attenuated substantially once adjusted for covariates, including comorbidity, suggesting that the bulk of the substantial increase in amputation risk associated with prior amputation was explained by the covariates included in our model, particularly comorbidity. Residual confounding is highly likely, wherein the measure of comorbidity used might not entirely represent the comorbidity load relevant to amputation risk.

The mortality rate among those who underwent amputations was high, with more than half of all those who underwent a major amputation and more than a third of those undergoing minor amputation dying within 3 years (Table 1). This death rate is comparable with those observed in other international contexts $[12,13]$ : for example, a study from the Netherlands observed a 1 year mortality of $44 \%$ and a 5 year mortality of $77 \%$ among individuals undergoing their first amputation [13]. This very high death rate clearly reflects the poor general health of those who require amputation, both in terms of the severity of their diabetes and the co-existence of other chronic conditions. Moreover, it is striking that $13 \%$ of those who did not undergo an amputation also died during the study follow-up period (up to the end of 2014). It is important to note that at least some of these individuals would have undergone a major (or minor) amputation if their prognosis had been better [37].

\section{Strengths and limitations}

Like many similarly constructed registers, the VDR is an imperfect measure of diabetes prevalence, though the algorithm used to define the population in the register has been iteratively improved over time [21]. If the VDR overestimates the size of the prevalent diabetic population in New Zealand, then it is possible that the absolute number of amputations attributed to diabetes in this study would also be an overestimate. This seems highly unlikely, as errors in cohort definition should be more common among those at the less severe end of the chronic disease spectrum, rather than those with strong (non-diabetic) risk factors for lower limb amputation. In other words, we are unlikely to have misclassified our cohort in a way that could meaningfully affect the results reported here.
The current study is limited to those variables available from national-level datasets in terms of understanding those factors that impact amputation risk. If available, data on sensory function (as a marker of neuropathy), information about diabetic foot care (such as access to foot care services) and glycaemic control may have at least partially explained some of the association between covariates (e.g. prior amputation) and subsequent amputation risk. For example, in their recent cohort study among those attending diabetes clinics in New Zealand, Robinson et al observed a $27 \%$ increase in risk of amputation for every $10 \mathrm{mmol} /$ mol increase in $\mathrm{HbA}_{1 \mathrm{c}}$ (adjusted HR 1.27, 95\% CI 1.24, 1.31) [14]. However, these glycaemic control data are currently unavailable at a national level in New Zealand, so while at least part of the adjusted associations presented here might be explained by poor glycaemic control, we do not have the data to confirm this.

Data from the VDR did not allow us to separate those with type 1 diabetes from those with type 2 diabetes. This would have been useful for cohort description, and could have been included as both an exposure of interest and covariate when considering the impact of other exposures such as age. This would also have allowed us to stratify analyses by diabetes type to examine whether the factors that most strongly influence amputation risk for type 1 diabetes differ from those that most strongly influence amputation risk for type 2 diabetes.

\section{Conclusions}

This study demonstrates the risk of major and minor amputation among a prevalent cohort of 217,207 people with diabetes in New Zealand. Those particularly at risk were men, indigenous Māori, those residing in the most-deprived areas and individuals with comorbidity. Previous amputation conferred a particularly high risk of subsequent amputation, but this may have less to do with the prior amputation per se than it does with the individual's underlying risk factors. Death was common among the total cohort, but particularly among those who underwent amputation, with more than half of those who underwent a major amputation dying within 3 years of their procedure. This is the first study to our knowledge that has investigated risk of amputation in a large, well-defined, national prevalent cohort of individuals with diabetes, strengthening the generalisability of our findings to the general population with diabetes.

Acknowledgements Data linking and extraction were performed by the National Collections staff at the New Zealand Ministry of Health. We thank I. Ratine and S. Ross from the Ministry of Health for their assistance with the data extraction. 
Data availability The data used for this study from the Virtual Diabetes Register and National Collections cannot be shared by the researchers because of ethical agreements. However, enquiries regarding accessing the data used for this study can be directed to the Ministry of Health's National Collections team.

Funding This study was partially funded by a University of Otago Wellington Dean's Grant (2016).

Duality of interest The authors declare that there is no duality of interest associated with this manuscript.

Contribution statement JG led study conception and design and the analysis and interpretation of data, drafted the manuscript and revised content based on feedback. JS assisted with study conception and design and interpretation of data and provided critical revision of drafts. SY assisted with interpretation of data and provided critical revision of drafts. DR assisted with interpretation of data and provided critical revision of drafts. DS assisted with study concept and design and interpretation of data and provided critical revision of drafts. All authors approved the final version of the manuscript. JG is responsible for the integrity of this work as a whole.

\section{References}

1. Reiber GE, Pecoraro RE, Koepsell TD (1992) Risk factors for amputation in patients with diabetes mellitus: a case-control study. Ann Intern Med 117:97-105

2. Holman N, Young RJ, Jeffcoate WJ (2012) Variation in the recorded incidence of amputation of the lower limb in England. Diabetologia 55:1919-1925

3. Miki E, Lu M, Lee ET, Keen H, Bennett PH, Russell D (2001) Risk factors, ethnic differences and mortality associated with lowerextremity gangrene and amputation in diabetes. The WHO multinational study of vascular disease in diabetes. Diabetologia 44(Suppl 2):S65-S71

4. Adler AI, Erqou S, Lima TAS, Robinson AHN (2010) Association between glycated haemoglobin and the risk of lower extremity amputation in patients with diabetes mellitus-review and meta-analysis. Diabetologia 53:840-849

5. Zhao W, Katzmarzyk PT, Horswell R et al (2013) HbAlc and lower-extremity amputation risk in low-income patients with diabetes. Diabetes Care 36:3591-3598

6. Zhou ZY, Liu YK, Chen HL, Yang HL, Liu F (2015) HbA1c and lower extremity amputation risk in patients with diabetes: a metaanalysis. Int J Low Extrem Wounds 14:168-177

7. Selby JV, Zhang D (1995) Risk factors for lower extremity amputation in persons with diabetes. Diabetes Care 18:509516

8. Holstein P, Ellitsgaard N, Olsen BB, Ellitsgaard V (2000) Decreasing incidence of major amputations in people with diabetes. Diabetologia 43:844-847

9. Tseng CL, Rajan M, Miller DR et al (2005) Use of administrative data to risk adjust amputation rates in a national cohort of medicareenrolled veterans with diabetes. Med Care 43:88-92

10. Shah SK, Bena JF, Allemang MT et al (2013) Lower extremity amputations: factors associated with mortality or contralateral amputation. Vasc Endovasc Surg 47:608-613
11. Izumi Y, Satterfield K, Lee S, Harkless LB (2006) Risk of reamputation in diabetic patients stratified by limb and level of amputation: a 10-year observation. Diabetes Care 29:566-570

12. Jupiter DC, Thorud JC, Buckley CJ, Shibuya N (2016) The impact of foot ulceration and amputation on mortality in diabetic patients. I: From ulceration to death, a systematic review. Int Wound J 13: 892-903

13. Fortington LV, Geertzen JH, van Netten JJ, Postema K, Rommers GM, Dijkstra PU (2013) Short and long term mortality rates after a lower limb amputation. Eur J Vasc Endovasc Surg 46:124-131

14. Robinson TE, Kenealy T, Garrett M, Bramley D, Drury PL, Elley CR (2016) Ethnicity and risk of lower limb amputation in people with Type 2 diabetes: a prospective cohort study. Diabet Med 33: $55-61$

15. Wirth RB, Marfin AA, Grau DW, Helgerson SD (1993) Prevalence and risk factors for diabetes and diabetes-related amputations in American Indians in Southern Arizona. Diabetes Care 16:354-356

16. Chen HF, Ho CA, Li CY (2006) Age and sex may significantly interact with diabetes on the risks of lower-extremity amputation and peripheral revascularization procedures: evidence from a cohort of a half-million diabetic patients. Diabetes Care 29:2409-2414

17. Amin L, Shah BR, Bierman AS et al (2014) Gender differences in the impact of poverty on health: disparities in risk of diabetesrelated amputation. Diabet Med 31:1410-1417

18. Moss SE, Klein R, Klein BEK (1992) The prevalence and incidence of lower extremity amputation in a diabetic population. Arch Intern Med 152:610-616

19. Bild DE, Selby JV, Sinnock P, Browner WS, Braveman P, Showstack JA (1989) Lower-extremity amputation in people with diabetes. Epidemiology and prevention. Diabetes Care 12:24-31

20. Jo EC, Drury PL (2015) Development of a Virtual Diabetes Register using information technology in New Zealand. Healthc Inform Res 21:49-55

21. Ministry of Health (2017) Virtual Diabetes Register. Available from www.health.govt.nz/our-work/diseases-and-conditions/diabetes/ about-diabetes/virtual-diabetes-register-vdr. Accessed 26 June 2017

22. Salmond C, Crampton P (2012) Development of New Zealand's Deprivation Index (NZDep) and its uptake as a national policy tool. Can J Public Health 103:S7-S11

23. Robson B, Purdie G, Cormack D (2010) Unequal impact II: Māori and non-Māori cancer statistics by deprivation and rural-urban status, 2002-2006. Ministry of Health, Wellington

24. Charlson M, Pompei P, Ales K, Mackenzie C (1987) A new method for classifying prognostic comorbidity in longitudinal studies: development and validation. J Chronic Dis 40:373-383

25. Quan H, Sundararajan V, Halfon P et al (2005) Coding algorithms for defining comorbidities in ICD-9-CM and ICD-10 administrative data. Med Care 43:1130-1139

26. Jeffcoate WJ, van Houtum WH (2004) Amputation as a marker of the quality of foot care in diabetes. Diabetologia 47:2051-2058

27. Rodrigues BT, Vangaveti VN, Malabu UH (2016) Prevalence and risk factors for diabetic lower limb amputation: a clinic-based case control study. J Diabetes Res 2016:5941957

28. Naqshbandi M, Harris SB, Esler JG, Antwi-Nsiah F (2008) Global complication rates of type 2 diabetes in Indigenous peoples: a comprehensive review. Diabetes Res Clin Pract 82:1-17

29. Chaturvedi N, Abbott CA, Whalley A, Widdows P, Leggetter SY, Boulton AJM (2002) Risk of diabetes-related amputation in South Asians vs. Europeans in the UK. Diabet Med 19:99-104

30. Lai YJ, Hu HY, Lin CH, Lee ST, Kuo SC, Chou P (2015) Incidence and risk factors of lower extremity amputations in people with type 2 diabetes in Taiwan, 2001-2010. J Diabetes 7:260-267 
31. Vestbo J, Hurd SS, Agustí AG et al (2013) Global strategy for the diagnosis, management, and prevention of chronic obstructive pulmonary disease GOLD executive summary. Am J Respir Crit Care Med 187:347-365

32. Selvin E, Erlinger TP (2004) Prevalence of and risk factors for peripheral arterial disease in the United States: results from the National Health and Nutrition Examination Survey, 1999-2000. Circulation 110:738-743

33. Armstrong DG, Boulton AJM, Bus SA (2017) Diabetic foot ulcers and their recurrence. N Engl J Med 376:2367-2375

34. Skoutas D, Papanas N, Georgiadis GS et al (2009) Risk factors for ipsilateral reamputation in patients with diabetic foot lesions. Int $\mathrm{J}$ Low Extrem Wounds 8:69-74
35. Armstrong DG, Lavery LA (1998) Plantar pressures are higher in diabetic patients following partial foot amputation. Ostomy Wound Manage 44:30-32

36. Lavery LA, Lavery DC, Quebedeax-Farnham TL (1995) Increased foot pressures after great toe amputation in diabetes. Diabetes Care 18:1460-1462

37. Jeffcoate WJ, Bus SA, Game FL, Hinchliffe RJ, Price PE, Schaper NC (2016) Reporting standards of studies and papers on the prevention and management of foot ulcers in diabetes: required details and markers of good quality. Lancet Diabetes Endocrinol 4:781788 